\title{
COMPACTABILIDADE DE UM LATOSSOLO AMARELO DISTROCOESO DOS TABULEIROS COSTEIROS DE ALAGOAS SOB DIFERENTES SISTEMAS DE MANEJO DA CANA-DE-AÇÚCAR(1)
}

\author{
Romero Falcão Bezerra de Vasconcelos ${ }^{(2)}$, José Ramon Barros \\ Cantalice $^{(3)}$, Geber Barbosa de Albuquerque Moura ${ }^{(3)}$, Mário Monteiro \\ Rolim $^{(2)}$ \& Carlos Eduardo de Vasconcelos Montenegro ${ }^{(4)}$
}

\begin{abstract}
RESUMO
A compactação do solo agrícola é um fenômeno de grande interesse para a Ciência do Solo. O estudo investigativo de diferentes sistemas de manejo visando encontrar aquele que degrade menos o solo é o objetivo maior da pesquisa sobre o comportamento mecânico do solo. De maneira geral, para um mesmo nível de energia de compactação, quanto maior o teor de matéria orgânica, menor a densidade máxima do solo e maior a umidade crítica de compactação; consequentemente, o risco de degradação física do solo se reduz. O objetivo deste trabalho experimental foi estudar a compactabilidade de um Latossolo Amarelo distrocoeso dos Tabuleiros Costeiros de Alagoas. Foram selecionadas três áreas em talhões sob cultivo da cana-de-açúcar, representativos de três sistemas de manejo adotados pela Usina Santa Clotilde. Os três sistemas de manejo investigados foram: uma área cultivada sob sistema de manejo irrigado (SMI), uma área sob sistema de manejo de fertirrigação com vinhaça (SMV) e uma área com aplicação de vinhaça + torta de filtro (SMVT). Os três sistemas de manejo foram implantados na safra 2003/2004, e a coleta de amostras do solo, realizada em fevereiro de 2007. Esses sistemas foram comparados entre si e em relação a uma testemunha-padrão, representada por uma mata nativa (MN). A densidade máxima do solo e a umidade crítica de compactação foram avaliadas nas profundidades de $0-0,20,0,20-0,40$ e $0,40-0,60 \mathrm{~m}$. Para avaliar a capacidade
\end{abstract}

\footnotetext{
(1) Parte da Tese de Doutorado do primeiro autor apresentada ao Programa de Pós-Graduação em Ciência do Solo da Universidade Federal Rural de Pernambuco - UFRPE. Recebido para publicação em 29 de março de 2011 e aprovado em 22 de dezembro de 2011.

(2) Professor do Departamento de Tecnologia Rural, Universidade Federal Rural de Pernambuco - UFRPE. Av. Dom. Manoel de Medeiros s/n, Dois Irmãos, CEP 52171-900 Recife (PE). E-mails: romerofalcao@hotmail.com; rolim@dtr.ufrpe.br

(3) Professor do Departamento de Agronomia, UFRPE. E-mails: cantalic@terra.com.br; geber@depa.ufrpe.br

(4) Graduando de Engenharia, UFPE. E-mail: dudu475@gmail.com
} 
dos resíduos da cana-de-açúcar em dissipar parte da energia compactante, foram realizados ensaios de Proctor, utilizando quatro níveis de energia de compactação. A avaliação da energia dissipada pelos resíduos foi feita em amostras deformadas apenas da profundidade de 0-0,20 m. O sistema de manejo irrigado (SMI), que não recebeu adição de resíduo da cana-de-açúcar, foi o que apresentou maior densidade máxima do solo e menor umidade crítica de compactação. Entre os diferentes sistemas de manejo cultivados com cana-de-açúcar, o mais eficiente na dissipação da energia compactante foi a área sob aplicação de vinhaça + torta de filtro, seguida da área sob sistema de manejo de fertirrigação com vinhaça, respectivamente com os valores de 54 e $41 \%$ de dissipação para o nível mais alto de energia de compactação. Verificou-se também que a dissipação da energia de compactação foi maior para os níveis de energia mais altos.

Termos de indexação: compactação do solo, matéria orgânica, dissipação de energia de compactação, ensaio de Proctor, resíduos da cana-de-açúcar.

\section{SUMMARY: COMPRESSIBILITY OF A DYSTROPHIC COHESIVE YELLOW LATOSOL IN THE COASTAL TABLELANDS OF ALAGOAS UNDER DIFFERENT SUGAR CANE MANAGEMENT SYSTEMS}

The compaction of agricultural soils is a phenomenon of great concern in Soil Science. The major goal of research on the mechanical behavior of soil is to identify the least degrading soil management systems. In general, at a same compaction level, the higher content of organic matter, the lower maximum density and higher critical moisture for compaction, which consequently decreases the risk of physical degradation of the soil. The purpose of this study was to study the compressibility of a dystrophic cohesive yellow latosol of the coastal plains of Alagoas. Three areas were selected in sugarcane plantations of the sugar mill Santa Clotilde, representing three management systems: sugarcane under irrigation (IMS), sugarcane under irrigation and vinasse application (SMV) and sugarcane with vinasse and filter cake application (SMVT). The three management systems were used in the 2003/2004 growing season and the soil sampled in February 2007. These systems were compared with each other and to a control area, represented by native forest (NF). The maximum soil density and critical moisture for compaction were evaluated in the layers $0-0.20,0.20-0.40$ and $0.40-0.60 \mathrm{~m}$. The ability of sugarcane residues to dissipate part of the compacting energy was assessed in Proctor tests at four levels of compression. The energy dissipated by crop residues was assessed in disturbed samples in the $0-0.20 \mathrm{~m}$ layer only. In the irrigated management system (IMS), without cane residue application, the maximum density was highest and critical soil moisture for compaction lowest. Of the different management systems, the most efficient in dissipating compression energy was the area under application of vinasse and filter cake, followed by the system of irrigation with vinasse, respectively, with respective values of 54 and $41 \%$ dissipation at the highest level of compression energy. It was also observed that the dissipation of compression energy was higher at higher energy levels.

Index terms: soil compaction, organic matter, compression energy dissipation, Proctor test, sugarcane residues.

\section{INTRODUÇÃO}

A compactação do solo é caracterizada pela redução do seu volume quando uma tensão externa lhe é aplicada. Segundo Collares et al. (2006), a compactação provoca alteração estrutural do solo devido à reorganização das partículas e de seus agregados, resultando em aumento da densidade e resistência do solo à penetração e da redução da macroporosidade, impedindo o crescimento e o desenvolvimento radicular das plantas. Raper (2005) considera que a compactação tem sido indicada como a principal causa da degradação física dos solos pela redução de seu espaço poroso. 
Barzegar et al. (2000) reportam numerosos estudos indicando que resíduos culturais, como palha de milho e de aveia, outros resíduos tenros e muito úmidos reduzem a compactabilidade do solo. De acordo com esses autores, o incremento da carga de compactação aumenta a suscetibilidade dos solos à compactação, e também concluíram que o resíduo da cana-de-açúcar foi efetivo na redução da compactação de solos coesos em conteúdos de água mais baixos do que 0,80 LP (LP = limite de plasticidade) no ensaio Proctor normal.

A matéria orgânica tem grande importância no comportamento mecânico do solo, principalmente quando ele é submetido à carga externa. Braída et al. (2006), avaliando o efeito da matéria orgânica sobre o comportamento mecânico de duas classes de solo, verificaram que o acúmulo de matéria orgânica proporcionado por diferentes sistemas de manejo reduziu a densidade máxima e aumentou a umidade crítica para compactação do solo, significando que ele se tornou mais difícil de ser compactado. Esses autores também observaram que a presença de palha picada sobre o solo, durante a realização do ensaio Proctor, dissipou parte da energia de compactação.

A influência da matéria orgânica no fenômeno de compactação é significativa, devido ao seu alto poder de absorção de água, dificultando a compactação (Silva et al., 1986; Dias Júnior \& Miranda, 2000), por proporcionar melhorias na estrutura do solo (Stone \& Ekwue, 1993) e apresentar menor densidade que a do solo (Ekwue \& Stone, 1995). Gupta et al. (1989) concluíram que os sistemas de manejo podem proporcionar um arranjamento mais compacto das partículas primárias e agregados decorrentes da compactação, possibilitando que ocorram alterações na sua capacidade de suporte de carga.

A matéria orgânica do solo repercute no valor da densidade máxima do solo, tanto pela melhoria na estabilidade estrutural do solo, como pelo fato de a densidade dela ser menor do que a dos sólidos do solo. Krzic et al. (2004), investigando o efeito da matéria orgânica do solo sobre o grau de compactação ou densidade relativa do solo, comprovaram que o incremento de $1 \%$ no teor de carbono orgânico reduz, independentemente da textura do solo, a densidade relativa máxima do solo em aproximadamente $11 \%$. Esses resultados confirmam a importância da adoção de sistemas de manejo que promovam o aumento no teor de matéria orgânica do solo.

Linstrom \& Voorhess (1994) consideram elevados os valores acima de $86 \%$ para grau de compactação. Valores superiores a esse limite crítico podem alterar negativamente a produtividade agrícola. Entretanto, o grau de compactação, que é a relação entre a densidade do solo e a densidade máxima do solo, isoladamente, não é suficiente para diagnosticar o efeito de atributos físicos do solo sobre as plantas, constituindo apenas um indicador de prováveis limitações ao desenvolvimento do sistema radicular. De acordo com Batey \& McKenzie (2006), existe uma variedade de critérios diagnósticos disponíveis para detectar a compactação no campo.

Dessa forma, o objetivo geral deste trabalho foi o estudo da compactabilidade de um Latossolo Amarelo distrocoeso dos Tabuleiros Costeiros cultivados com cana-de-açúcar, tendo como objetivos específicos: avaliar a influência de três sistemas de manejo da cana-de-açúcar sobre os parâmetros de compactabilidade, bem como a capacidade da matéria orgânica em dissipar parte da energia de compactação produzida pelo tráfego de máquinas.

\section{MATERIAL E MÉTODOS}

O experimento foi realizado na Fazenda Pau Amarelo, de propriedade da Usina Santa Clotilde S/A, compreendida entre as coordenadas $9^{\circ} 27^{\prime} 31^{\prime \prime} \mathrm{S}$,

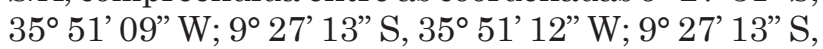
$35^{\circ} 50^{\prime} 56^{\prime \prime} \mathrm{W}$; e $9^{\circ} 27^{\prime} 32^{\prime \prime} \mathrm{S}, 35^{\circ} 50^{\prime} 54^{\prime}$ 'W, que têm como referência horizontal o datum WGS84. A Fazenda Pau Amarelo está localizada no município de Rio Largo, litoral do Estado de Alagoas; tem clima As, tropical chuvoso com verão seco, segundo a classificação de Köppen \& Geiger (1928). A precipitação pluvial média anual é de $1.752 \mathrm{~mm}$, e a temperatura média anual, de $29{ }^{\circ} \mathrm{C}$.

O solo estudado foi classificado como Latossolo Amarelo distrocoeso (Embrapa, 2006), relevo plano, textura média/argilosa. O solo é derivado do grupo Barreiras, o qual é formado por sedimentos arenoargilosos, com a fração areia constituída basicamente de quartzo e a fração argila de caulinita, com baixos teores de óxidos de ferro (Jacomine et al., 1975).

Foram selecionadas áreas em talhões de produção com a cultura da cana-de-açúcar, sendo objeto de pesquisa três áreas, representativas de três sistemas de manejo adotados pela unidade sucroalcooleira. Dessa forma, os tratamentos avaliados foram uma área cultivada sob sistema de manejo irrigado (SMI), uma área cultivada sob sistema de manejo de fertirrigação com vinhaça (SMV) e uma área cultivada com a aplicação de vinhaça + torta de filtro (SMVT). As três áreas são exploradas com cana-de-açúcar há mais de 20 anos, sendo o relevo plano a suave ondulado, característico dos solos dos Tabuleiros Costeiros. Esses sistemas de manejo foram comparados entre si e em relação a uma testemunha-padrão, reproduzida por solo em condições naturais (floresta nativa). Os três sistemas de manejo estudados na 
pesquisa experimental foram adotados na unidade sucroalcooleira desde a safra 2003/2004. A coleta do solo foi realizada no mês de fevereiro de 2007.

Durante o período do trabalho experimental foi executado um rígido controle de tráfego de máquinas nas três áreas cultivadas com cana-deaçúcar. O manejo cultural no plantio e na renovação do canavial e a mobilização mecânica do solo são descritos a seguir e foram iguais nas três áreas estudadas. O preparo periódico do solo foi feito com o uso de subsoladores de três elementos ativos de hastes parabólicas, cuja forma de mobilização é constituída por cortes verticais com intensa vibração no interior do solo. Em seguida, usaram-se grades de discos de dupla ação, com a finalidade de destruir a socaria velha e, ao mesmo tempo, provocar o deslocamento horizontal do solo, deixando-o em condições para o plantio. Finalmente, foram abertos sulcos de aproximadamente $0,25 \mathrm{~m}$ de profundidade. O plantio foi realizado manualmente, sendo colocadas no fundo do sulco as canas-sementes, que posteriormente foram cobertas com solo. Em todas as três áreas, a cana-de-açúcar foi queimada antes da operação de colheita. Na colheita, a cana foi cortada manualmente e transportada para o caminhão por carregadoras convencionais.

$\mathrm{Na}$ área cultivada sob SMI foi aplicada uma lâmina de $120 \mathrm{~mm}$ de água por ciclo da cultura. A adubação química da cana-planta foi realizada de acordo com a análise do solo, sendo aplicados 40, 120 e $90 \mathrm{~kg} \mathrm{ha}^{-1}$ de $\mathrm{N}, \mathrm{P}_{2} \mathrm{O}_{5}$ e $\mathrm{K}_{2} \mathrm{O}$, respectivamente, e, na cana-soca, 80, 50 e $90 \mathrm{~kg} \mathrm{ha}^{-1}$ de $\mathrm{N}, \mathrm{P}_{2} \mathrm{O}_{5}$ e $\mathrm{K}_{2} \mathrm{O}$, respectivamente.

$\mathrm{Na}$ área cultivada com SMV foram aplicados, por ciclo de cultura, $300 \mathrm{~m}^{3} \mathrm{ha}^{-1}$ de vinhaça diluída, por aspersão. Na adubação química da cana-planta foram aplicados $40 \mathrm{~kg} \mathrm{ha}^{-1}$ de $\mathrm{N}$ e na cana-soca, $80 \mathrm{~kg} \mathrm{ha}^{-1}$ de $\mathrm{N}$.

$\mathrm{Na}$ área cultivada de acordo com o SMVT foram aplicados $30 \mathrm{Mg} \mathrm{ha}^{-1}$ de torta de filtro associada com vinhaça, por aspersão. Na adubação química da cana-planta foram aplicados $40 \mathrm{~kg} \mathrm{ha}^{-1}$ de $\mathrm{N} \mathrm{e}$, na cana-soca, $80 \mathrm{~kg} \mathrm{ha}^{-1}$ de $\mathrm{N}$.

As amostras de solo deformadas foram coletadas ao acaso, nas áreas com os diferentes sistemas de manejo, nas profundidades de $0-0,20,0,20-0,40$ e $0,40-0,60 \mathrm{~m}$, sendo retiradas num ponto situado nas entrelinhas de plantio da cana-de-açúcar.

$\mathrm{Na}$ análise granulométrica empregou-se o método do densímetro de Boyoucos, e na dispersão física do solo utilizou-se um agitador mecânico durante 15 min e, como dispersante químico, o calgon (Embrapa, 1997). A densidade das partículas foi determinada pelo método do balão volumétrico, e a densidade do solo, obtida com anel volumétrico (Embrapa, 1997).
Para avaliar o teor de matéria orgânica do solo, determinou-se o teor de carbono orgânico, em cada uma das amostras, conforme método descrito por Embrapa (1997).

A estabilidade dos agregados foi determinada por via úmida, sendo as amostras coletadas ao acaso, com auxílio de enxadão e pá de corte. $\mathrm{Na}$ separação de agregados por via úmida, adotou-se o procedimento descrito por Kemper \& Rosenau (1986), por meio de peneiramento em água, após préumedecimento lento dos agregados, por capilaridade. Os agregados assim preparados foram transferidos para dois conjuntos de cinco peneiras de 3,$35 ; 2,00$; 1,00; 0,50; e 0,25 mm de diâmetro de malha, sendo submetidos à agitação vertical (42 ciclos por minuto) durante $15 \mathrm{~min}$, imersos em recipiente com água. $\mathrm{O}$ solo retido em cada peneira foi acondicionado em recipientes de alumínio para secagem em estufa a $105{ }^{\circ} \mathrm{C}$, durante $24 \mathrm{~h}$. Em seguida, pesou-se e calculou-se a massa e a percentagem de agregados estáveis em cada uma das classes de diâmetro de agregados.

O limite de plasticidade (LP) corresponde ao teor de umidade no qual o solo começa a fissurar, quando rolado, para formar pequenos cilindros de dimensões padronizadas. Foi determinado de acordo com a ABNT (1984).

O ensaio Proctor Normal, descrito por Proctor (1933), foi realizado com o emprego de um equipamento denominado cilindro de Proctor, marca Contenco, em conformidade com o método descrito pela norma NBR 7182 (ABNT, 1986). Todas as amostras deformadas dos solos dos sistemas de manejo foram coletadas nas três profundidades estudadas. A amostra de solo foi compactada em um anel metálico de aproximadamente $1.000 \mathrm{~cm}^{3}$, em três camadas, cada uma recebendo 26 golpes de um soquete de $2,5 \mathrm{~kg}$, caindo de uma altura de $30,5 \mathrm{~cm}$, correspondendo a uma energia de $60,2 \mathrm{~kJ} \mathrm{~m}^{-3}$.

Para construção da curva de compactação, o solo foi compactado em cinco teores de umidade, com intervalos de $0,02 \mathrm{~kg} \mathrm{~kg}^{-1}$ de umidade, de modo que o terceiro ponto da curva de compactação ficasse próximo da umidade crítica de compactação $\left(\mathrm{U}_{\mathrm{cc}}\right)$. Após cada compactação, foram retiradas duas amostras do corpo de prova (topo e fundo), para determinação do teor de água do solo. Plotando os valores de umidade versus densidade do solo, construiu-se a curva de compactação.

Com o objetivo de mensurar a capacidade dos sistemas de manejo em absorver parte da energia de compactação, realizou-se o ensaio de Proctor, usando-se níveis de energia de 24,$1 ; 36,1 ; 60,2$; e $84,3 \mathrm{~kJ} \mathrm{~m}^{-3}$, correspondendo, respectivamente, a 10 , 15, 25 e 35 golpes (Braida et al., 2006).

Nessa investigação foram utilizadas amostras deformadas apenas da profundidade de $0-0,20 \mathrm{~m}$, 
e o sistema de manejo da área cultivada sob irrigação (SMi) foi onsiderado testemunha, pois não recebeu resíduos da cana-de-açúcar. Com base nos resultados obtidos para o $\mathrm{SMi}$, ajustou-se a seguinte equação:

$$
\mathrm{D}_{\text {Smax }}=\mathrm{a}+\mathrm{b}\left(\mathrm{E}_{\mathrm{c}}\right)+\mathrm{c}\left(\mathrm{E}_{\mathrm{c}}\right)^{2}
$$

em que $\mathrm{D}_{\text {Smax }}=$ densidade máxima do solo $\left(\mathrm{Mg} \mathrm{m}^{-3}\right)$; $\mathrm{E}_{\mathrm{c}}=$ energia de compactação $\left(\mathrm{kJ} \mathrm{m}^{-3}\right) ; \mathrm{a}, \mathrm{b}$ e $\mathrm{c}=$ coeficientes de ajuste.

Com base na equação 1 , que descreve a relação entre densidade máxima do solo e energia de compactação, estimou-se a energia de compactação correspondente, a partir dos dados de densidades dos sistemas de manejo descritos.

Baseando-se na energia de compactação empregada nos diferentes sistemas de manejo e na energia equivalente correspondente, foi possível predizer a energia dissipada por esses sistemas.

Os dados foram submetidos à análise de variância, e as médias, comparadas pelo teste de Tukey a $5 \%$, utilizando o programa Saeg (2005). Estudos de correlações foram executados entre algumas variáveis medidas, empregando o programa Statistica 6.0 (Statsoft..., 2001).

\section{RESULTADOS E DISCUSSÃO}

\section{Avaliação de parâmetros da curva de compac- tação e dissipação de energia}

As curvas de compactação, obtidas por meio do ensaio de Proctor normal para os sistemas de manejo, nas três profundidades investigadas, são apresentadas na figura 1 . As curvas de compactação para os sistemas de manejo com cana-de-açúcar, em comparação ao solo de mata nativa, apresentaram deslocamento à esquerda e para cima nas três profundidades, resultando em maiores valores de densidade máxima do solo e menores de umidade crítica de compactação.

Analisando separadamente os sistemas de manejo com cana-de-açúcar, verificou-se que o solo da área sob irrigação com Dmáx $=1,808 \mathrm{Mg} \mathrm{m}^{-3}$ e Ucc $=0,145 \mathrm{~kg} \mathrm{~kg}^{-1}$ apresentou valores para os parâmetros de compactabilidade do solo estatisticamente diferentes quando comparado aos dois outros sistemas de manejo de área cultivada (Quadro 1). Esse comportamento está associado à redução do carbono orgânico total, principalmente na camada de $0-0,20 \mathrm{~m}$.

As áreas sob cultivo com cana-de-açúcar com aplicação de vinhaça + torta de filtro e de vinhaça apresentaram comportamento mais próximo das condições do solo sob mata nativa. Alterações nos parâmetros de compactabilidade do solo, devido às reduções nos teores de matéria orgânica, foram observadas por diversos pesquisadores (Barzegar et al., 2000; Braida et al., 2006; Silva et al., 2006). Geralmente, para um mesmo nível de energia de compactação, associa-se maior teor de matéria orgânica do solo com menor valor de densidade máxima do solo e maior valor de umidade crítica.

O quadro 2 apresenta as relações entre o limite de plasticidade e a umidade crítica de compactação. A análise evidencia o efeito da adição de resíduos pelos manejos culturais da cana-de-açúcar sobre o comportamento mecânico do solo, com destaque para aquele sob mata nativa, em que a umidade crítica de compactação $\left(0,24 \mathrm{~kg} \mathrm{~kg}^{-1}\right)$ supera o limite de plasticidade $\left(0,22 \mathrm{~kg} \mathrm{~kg}^{-1}\right)$, na profundidade de

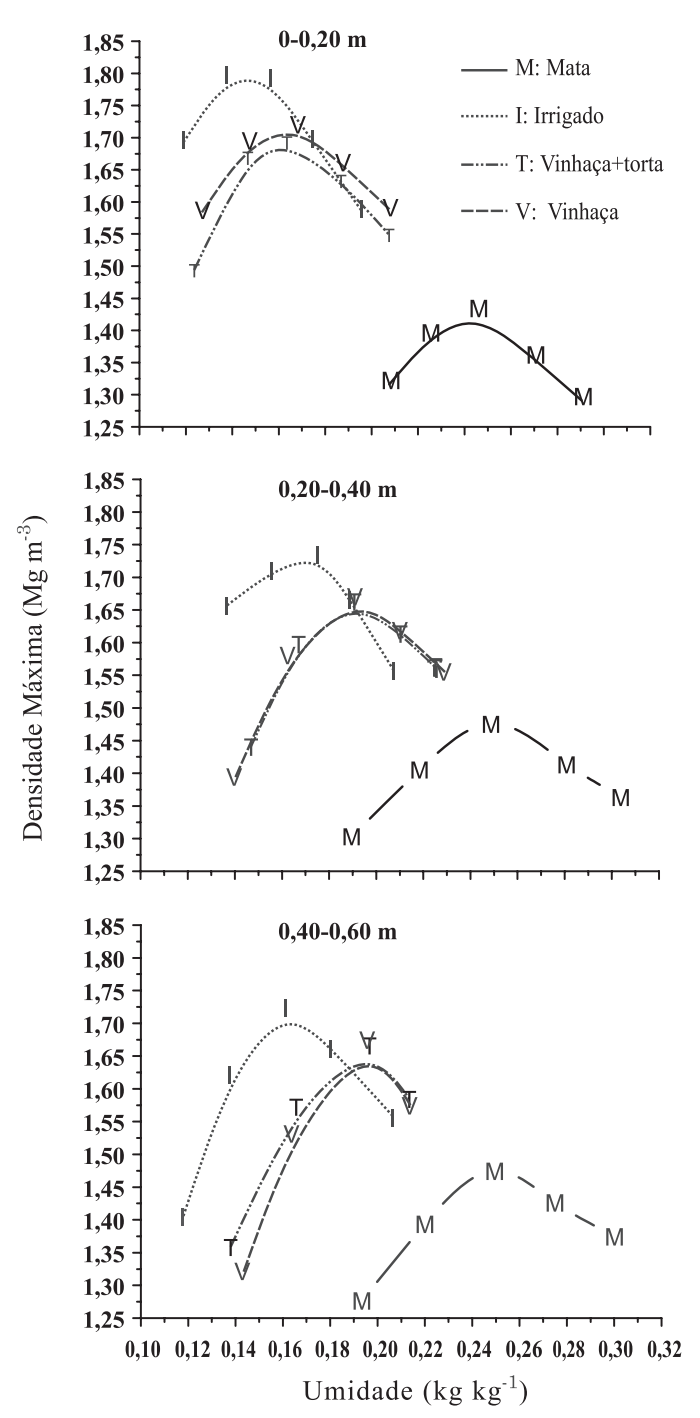

Figura 1. Curvas de compactação do solo dos quatro sistemas de manejo, nas três profundidades estudadas. 
0-0,20 m; para as camadas subsuperficiais $(0,20-$ 0,40 e $0,40-0,60 \mathrm{~m}$ ) o comportamento foi semelhante. Quanto aos sistemas de manejo sob cultivo com cana-de-açúcar, verifica-se que a umidade crítica

Quadro 1. Umidade crítica de compactação (Ucc) e densidade máxima do solo (Dmáx) obtidas pelo ensaio Proctor Normal e teor de carbono orgânico total (COT), para os diferentes sistemas de manejo e profundidades

\begin{tabular}{rcccc}
\hline \multirow{5}{*}{ Camada } & \multicolumn{4}{c}{ Sistema de manejo } \\
\cline { 2 - 5 } & SMI & MN & SMVT & SMV \\
\hline m & \multicolumn{5}{c}{ Ucc $\left(\mathrm{kg} \mathrm{kg}^{-1}\right)$} \\
$0-0,20$ & $0,145 \mathrm{Cc}$ & $0,242 \mathrm{Aa}$ & $0,160 \mathrm{Bb}$ & $0,162 \mathrm{Bb}$ \\
$0,20-0,40$ & $0,170 \mathrm{Ca}$ & $0,249 \mathrm{Aa}$ & $0,185 \mathrm{Ba}$ & $0,189 \mathrm{Ba}$ \\
$0,40-0,60$ & $0,157 \mathrm{Cb}$ & $0,250 \mathrm{Aa}$ & $0,191 \mathrm{Ba}$ & $0,191 \mathrm{Ba}$ \\
& \multicolumn{5}{c}{ Dmáx $\left(\mathrm{Mg} \mathrm{m}{ }^{-3}\right)$} \\
$0-0,20$ & $1,808 \mathrm{Aa}$ & $1,430 \mathrm{Ca}$ & $1,695 \mathrm{Ba}$ & $1,729 \mathrm{Bab}$ \\
$0,20-0,40$ & $1,742 \mathrm{Ab}$ & $1,478 \mathrm{Ca}$ & $1,670 \mathrm{Ba}$ & $1,672 \mathrm{Bb}$ \\
$0,40-0,60$ & $1,734 \mathrm{Ab}$ & $1,475 \mathrm{Ba}$ & $1,681 \mathrm{Aa}$ & $1,687 \mathrm{Aab}$ \\
& \multicolumn{5}{c}{ Carbono orgânico total ( \%) } \\
$0,0,20$ & $1,26 \mathrm{Ca}$ & $3,05 \mathrm{Aa}$ & $1,59 \mathrm{Ba}$ & $1,51 \mathrm{Ba}$ \\
$0,20-0,40$ & $0,87 \mathrm{Bb}$ & $1,85 \mathrm{Ab}$ & $0,85 \mathrm{Bb}$ & $0,68 \mathrm{Bb}$ \\
$0,40-0,60$ & $0,80 \mathrm{Bb}$ & $1,23 \mathrm{Ac}$ & $0,69 \mathrm{Bb}$ & $0,60 \mathrm{Bb}$ \\
\hline
\end{tabular}

Letras maiúsculas iguais na mesma linha e minúsculas iguais na mesma coluna não diferem significativamente pelo teste de Tukey (5\%). Média de três repetições. SMI: área irrigada; MN: mata nativa; SMVT: área de vinhaça + torta; SMV: área de vinhaça.

Quadro 2. Limite de plasticidade (LP), umidade correspondente a $90 \%$ do limite de plasticidade e umidade crítica de compactação (Ucc), considerando os sistemas de manejo e profundidades

\begin{tabular}{clllll}
\hline \multirow{2}{*}{ Profundidade } & Limite & \multicolumn{5}{c}{ Sistema de manejo } \\
\cline { 3 - 6 } & & SMI & MN & SMVT & SMV \\
\cline { 3 - 6 } m & & \multicolumn{4}{c}{$\mathrm{kg} \mathrm{kg}^{-1}$} \\
\cline { 3 - 6 } $0-0,20$ & LP & 0,18 & 0,22 & 0,19 & 0,19 \\
& $90 \% \mathrm{LP}$ & 0,16 & 0,20 & 0,17 & 0,17 \\
& Ucc & 0,15 & 0,24 & 0,16 & 0,16 \\
$0,20-0,40$ & LP & 0,19 & 0,23 & 0,21 & 0,23 \\
& $90 \% \mathrm{LP}$ & 0,17 & 0,21 & 0,19 & 0,21 \\
& Ucc & 0,17 & 0,25 & 0,19 & 0,19 \\
& LP & 0,20 & 0,24 & 0,22 & 0,24 \\
& $90 \% \mathrm{LP}$ & 0,18 & 0,22 & 0,20 & 0,22 \\
& Ucc 0,60 & 0,16 & 0,25 & 0,19 & 0,19 \\
\hline
\end{tabular}

SMI: área irrigada; MN: mata nativa; SMVT: área de vinhaça + torta; SMV: área de vinhaça. de compactação situa-se um pouco abaixo do limite de plasticidade nas três profundidades. O solo, nas condições da mata nativa, apresenta baixo risco de compactação. A tendência da relação Ucc/LP, no solo estudado, foi semelhante à observada por Ojeniyi \& Dexter (1979), que preconizam uma razão Ucc/LP em torno de 0,90 , sugerindo que a Ucc seja estimada próxima de $90 \%$ do LP.

Segundo Camargo \& Alleoni (1997), a grande capacidade de retenção de água da matéria orgânica reduz a quantidade de água entre as partículas minerais do solo, sendo necessário maior quantidade de água para que a densidade máxima do solo seja alcançada.

O melhor momento para realizar o preparo periódico do solo é aquele de consistência friável, que constitui a faixa ótima de umidade para mobilização mecânica do solo, pela facilidade de esboroamento quando ele é submetido às pressões provocadas pelas ferramentas ativas dos implementos agrícolas. Entretanto, a umidade crítica de compactação do solo também encontra-se nessa faixa de umidade.

Na figura 2 observa-se que a densidade máxima do solo apresentou correlação significativa e negativa $(\mathrm{r}=-0,7429)$ com o diâmetro médio ponderado úmido $(\mathrm{DMPu})$, comprovando forte relação funcional entre essas duas variáveis, no solo pesquisado. Esse fato reforça a importância da adoção de sistemas de manejo que promovam o aumento no teor de matéria orgânica do solo. A densidade máxima do solo foi influenciada pelo diâmetro médio ponderado dos agregados, tornando-se menor à medida que 0 diâmetro dos agregados aumentou.

$\mathrm{Na}$ figura 3 , pode-se verificar que a densidade máxima do solo (Dmáx) apresentou, também, correlação significativa $(\mathrm{p}<0,01)$ e positiva $(\mathrm{r}=$ 0,9074 ) com a densidade do solo (Ds), evidenciando forte relação funcional entre as duas variáveis para o solo investigado.

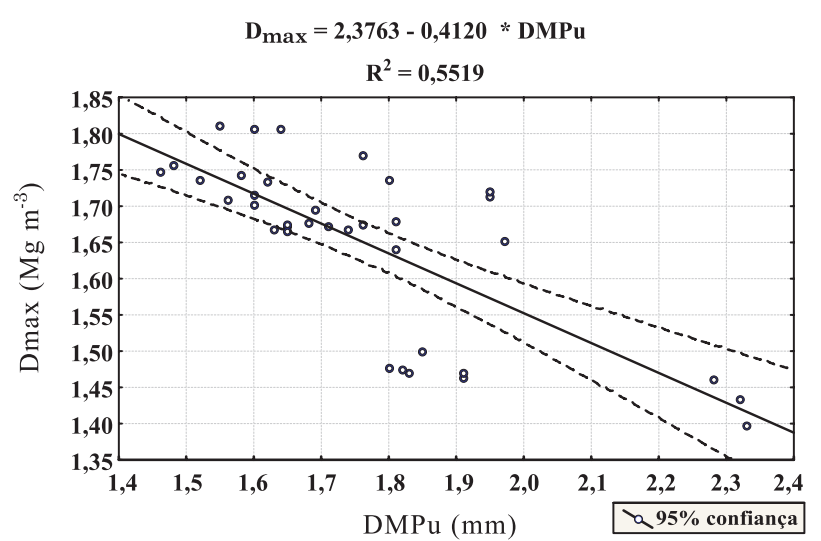

Figura 2. Relação entre a densidade máxima do solo (Dmáx) e o diâmetro médio ponderado dos agregados via úmida (DMPu). 


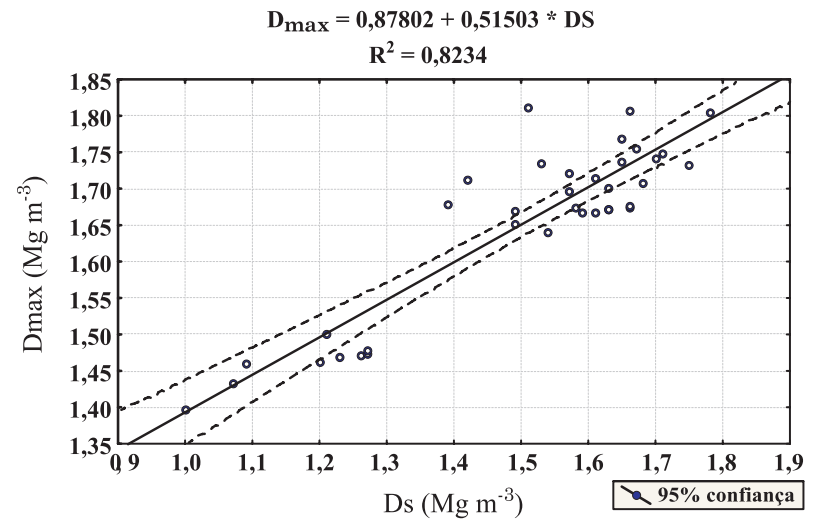

Figura 3. Relação entre a densidade máxima do solo (Dmáx) e a densidade do solo (Ds).

A figura 4 expressa o resultado da análise de regressão múltipla linear da densidade máxima do solo (Dmáx), em função da densidade do solo (Ds) e do diâmetro médio ponderado dos agregados via úmida (DMPu). A estimativa de Dmáx baseou-se no cálculo, por meio de modelo linear ajustado, com intervalo de confiança a $95 \%$ para o valor estimado. Nesse modelo de predição para a densidade máxima do solo, obteve-se $\mathrm{R}^{2}=0,8234$, que corresponde a um coeficiente de correlação da ordem de $\mathrm{r}=0,9074$ entre os valores observados e estimados. Além disso, é alcançado elevado nível de significância estatística, pois a probabilidade de erro (do tipo I) é p $<0,00001$.

A Dmáx cresceu com o aumento da energia de compactação, enquanto a Ucc apresentou comportamento inverso (Quadro 3). A área sob irrigação apresentou maior incremento (25\%) no valor da densidade máxima do solo e maior redução (38 \%) da umidade crítica, na média, em comparação com o solo sob mata nativa. Esse comportamento pode ser explicado pelo fato de ser o sistema de manejo que proporcionou menor aporte de matéria

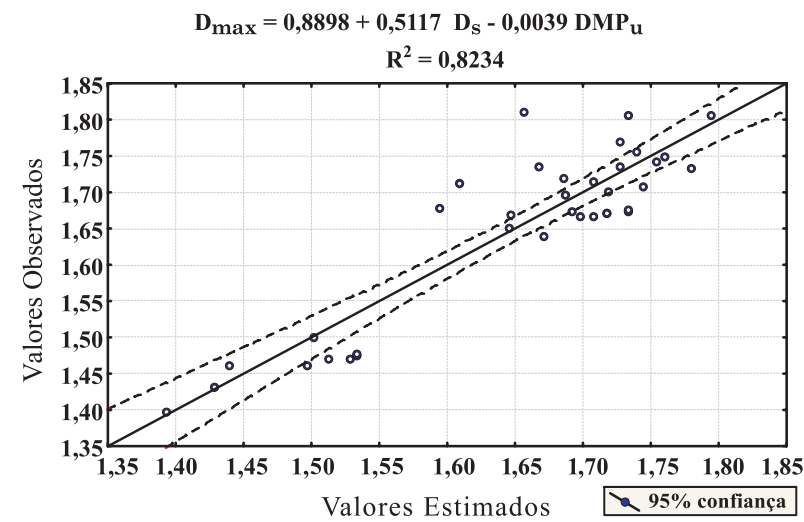

Figura 4. Modelo de densidade máxima do solo (Dmáx) - valores estimados contra valores observados. orgânica. As áreas de vinhaça + torta de filtro e de vinhaça apresentaram aumentos da ordem de 18 e $20 \%$ e reduções de 30 e $32 \%$, respectivamente, nos valores médios da variável Dmáx e Ucc, em comparação com o solo sob mata nativa. Esses resultados estão de acordo com os encontrados por Braida et al. (2006), que concluíram que o acúmulo de matéria orgânica, devido a diferentes sistemas de manejo, reduziu a Dmáx e aumentou a Ucc do solo, significando que este se tornou de mais difícil compactação. Acharya \& Sharna (1994) e Dao (1996), investigando o efeito da matéria orgânica sobre a densidade do solo, concluíram que ela foi menor quando sobre ele havia resíduos vegetais. Essas conclusões ratificam os resultados aqui apresentados.

A Dmáx cresceu com o aumento da energia de compactação para o sistema de manejo sob irrigação (Figura 5), com função quadrática $\left(\mathrm{R}^{2}=0,9862\right)$. $\mathrm{O}$ sistema de manejo irrigado foi utilizado como padrão na construção da curva de Dmáx em função dos níveis de energia de compactação, pelo fato de ele não ter recebido resíduos da cana-de-açúcar.

Com base nos valores de Dmáx obtidos nos demais sistemas de manejo em função de diferentes níveis de energia de compactação e empregandose a equação que descreve a relação Dmáx vs Ec

Quadro 3. Umidade crítica de compactação (Ucc) e densidade máxima do solo (Dmáx) obtidos pelo ensaio de Proctor para os diferentes sistemas de manejo e níveis de energia, na profundidade de 0 a $0,20 \mathrm{~m}$

\begin{tabular}{|c|c|c|c|c|c|}
\hline \multirow{2}{*}{$\begin{array}{c}\text { Niveis } \\
\text { de } \\
\text { energia }\end{array}$} & \multicolumn{5}{|c|}{ Sistema de manejo } \\
\hline & SMI & MN & SMVT & SMV & Média \\
\hline $\mathrm{kJ} \mathrm{m} \mathrm{m}^{-3}$ & \multicolumn{5}{|c|}{ Ucc $\left(\mathrm{kg} \mathrm{kg}^{-1}\right)$} \\
\hline 24,1 & $0,180 \mathrm{Ca}$ & $0,278 \mathrm{Aa}$ & $0,205 \mathrm{Ba}$ & $0,186 \mathrm{Ca}$ & \\
\hline 36,1 & $0,151 \mathrm{Cb}$ & $0,260 \mathrm{Ab}$ & $0,176 \mathrm{Bb}$ & $0,173 \mathrm{Bb}$ & \\
\hline 60,2 & $0,145 \mathrm{Cb}$ & $0,242 \mathrm{Ac}$ & $0,160 \mathrm{Bc}$ & $0,162 \mathrm{Bb}$ & \\
\hline \multirow[t]{2}{*}{84,3} & $0,132 \mathrm{Cc}$ & 0,207Ad & $0,152 \mathrm{Bc}$ & $0,145 \mathrm{Bc}$ & \\
\hline & \multicolumn{5}{|c|}{$\operatorname{Dmax}\left(\mathrm{Mg} \mathrm{m}^{-3}\right)$} \\
\hline 24,1 & 1,680 & 1,360 & 1,620 & 1,640 & $1,575 \mathrm{a}$ \\
\hline 36,1 & 1,735 & 1,376 & 1,634 & 1,664 & $1,602 \mathrm{~b}$ \\
\hline 60,2 & 1,808 & 1,430 & 1,695 & 1,729 & $1,665 \mathrm{c}$ \\
\hline 84,3 & 1,861 & 1,512 & 1,743 & 1,786 & $1,725 \mathrm{~d}$ \\
\hline Média & $1,771 \mathrm{~A}$ & 1,419B & $1,673 \mathrm{C}$ & $1,705 \mathrm{D}$ & \\
\hline
\end{tabular}

Letras maiúsculas iguais na mesma linha e minúsculas iguais na mesma coluna não diferem significativamente pelo teste de Tukey (5\%). Média de três repetições. SMI: área irrigada; MN: mata nativa; SMVT: área de vinhaça + torta; SMV: área de vinhaça. 
(Figura 5), estimou-se a energia de compactação equivalente. Posteriormente, também foi estimada a percentagem de energia dissipada pelos sistemas de manejo (Quadro 4).

Entre os sistemas de manejo cultivados com cana-de-açúcar, o mais eficiente na dissipação da

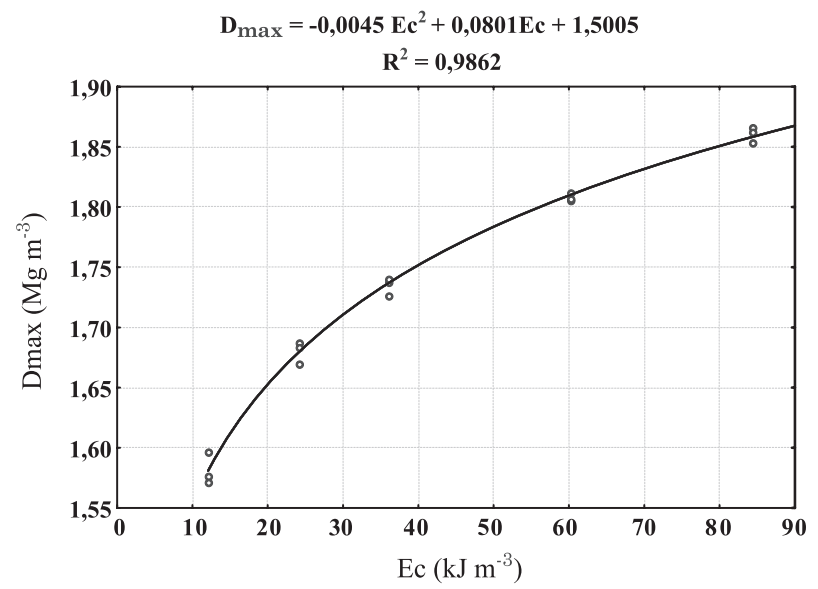

Figura 5. Densidade máxima do solo (área irrigada) em função de diferentes níveis de energia de compactação adotados no ensaio Proctor, na profundidade de $\mathbf{0}-0,20 \mathrm{~m}$.

Quadro 4. Densidade máxima do solo (Dmáx), energia equivalente (Ee) e energia dissipada (Ed) em função da energia de compactação (Ec) e dos sistemas de manejo no ensaio Proctor (médias de três repetições), na camada de 0 a $0,20 \mathrm{~m}$

\begin{tabular}{|c|c|c|c|c|c|}
\hline Ec & Manejo & Dmáx & $-\mathbf{E e}$ & 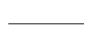 & Ed \\
\hline $\mathrm{kJ} \mathrm{m}^{-3}$ & & $\mathrm{Mg} \mathrm{m}^{-3}$ & $\mathrm{~kJ} \mathrm{~m}^{-3}$ & {[} & 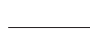 \\
\hline \multirow{3}{*}{84,3} & $\begin{array}{l}\text { SMI } \\
\text { MN }\end{array}$ & $\begin{array}{l}1,861 \\
1,512\end{array}$ & $\begin{array}{r}84,3 \\
-.\end{array}$ & $\begin{array}{r}100,0 \\
-\end{array}$ & 0,00 \\
\hline & SMVT & 1,743 & 38,7 & 45,9 & 54,10 \\
\hline & SMV & 1,786 & 49,3 & 58,5 & 41,50 \\
\hline \multirow{4}{*}{60,2} & SMI & 1,808 & 60,2 & 100,0 & 0,00 \\
\hline & $\mathrm{MN}$ & 1,430 & -- & -- & -- \\
\hline & SMVT & 1,695 & 29,0 & 48,2 & 51,80 \\
\hline & SMV & 1,729 & 35,7 & 59,3 & 40,70 \\
\hline \multirow{4}{*}{36,1} & SMI & 1,735 & 36,1 & 100,0 & 0,00 \\
\hline & $\mathrm{MN}$ & 1,376 & -. & -. & -- \\
\hline & SMVT & 1,634 & 18,6 & 51,5 & 48,50 \\
\hline & SMV & 1,664 & 23,5 & 65,1 & 34,90 \\
\hline \multirow{4}{*}{24,1} & SMI & 1,680 & 24,1 & 100,0 & 0,00 \\
\hline & MN & 1,360 & & & \\
\hline & SMVT & 1,620 & 16,4 & 68,1 & 31,90 \\
\hline & SMY & 1,640 & 19,6 & 81,3 & 18,70 \\
\hline 12,1 & SMI & 1,582 & 12,1 & 100,0 & 0,00 \\
\hline
\end{tabular}

SMI: área irrigada; MN: mata nativa; SMVT: área de vinhaça + torta; SMV: área de vinhaça. energia de compactação foi o cultivado sob aplicação de vinhaça+torta de filtro, seguido da área sob aplicação de vinhaça (Quadro 4). Apesar da prática cultural da queima da cana-de-açúcar antes da colheita, ficou confirmado que o acúmulo de matéria orgânica proporcionado pelos dois sistemas de manejo supracitados foi responsável pela dissipação de parte da energia de compactação, significando que o solo tornou-se mais difícil de ser compactado. Constatou-se, também, que a dissipação da energia de compactação é maior para os níveis de energia de compactação mais altos.

\section{CONCLUSÕES}

1. O sistema de manejo irrigado (SMI), que não recebeu adição de resíduo da cana-de-açúcar, foi o que apresentou maior densidade máxima do solo e menor umidade crítica de compactação.

2. Entre os sistemas de manejo do solo cultivado com cana-de-açúcar, o mais eficiente na dissipação de energia compactante foi o SMVT, seguido do SMV, com os valores de 54 e $41 \%$ de dissipação, respectivamente, para o nível mais alto de energia de compactação. A dissipação da energia de compactação foi maior para os níveis de energia mais elevados.

\section{LITERATURA CITADA}

ACHARYA, C.L. \& SHARMA, P.D. Tillage and mulch effects on soil physical environment root growth nutriente uptake and yield of maize and wheat on an Alfisol in north-west India. Soil Tillage Res., 32:291-302, 1994.

ASSOCIAÇÃO BRASILEIRA DE NORMAS TÉCNICAS - ABNT. NBR 7180: Solo: Determinação do limite de plasticidade. Rio de Janeiro, 1984. 3p.

ASSOCIAÇÃO BRASILEIRA DE NORMAS TÉCNICAS - ABNT. NBR 7182: Solo: Ensaio de compactação. Rio de Janeiro, 1986. 10p.

BARZEGAR, A.R.; ASOODAR, M.A. \& ANSARI, M. Effectiveness of sugar-cane residue incorporation at different water contents and the Proctor compaction loads in reducing soil compactibility. Soil Tillage Res., 57:167-172, 2000.

BATEY, T. \& McKENZIE, D.C. Soil compaction: Identification directly in the field. Brit. Soc. Sci. Soil Use Manag., 22:123-131, 2006.

BRAIDA, J.A.; REICHERT, J.M.; VEIGA, M. \& REINERT, D.J. Resíduos vegetais na superfície e carbono orgânico do solo e suas relações com a densidade máxima obtida no ensaio Proctor. R. Bras. Ci. Solo, 30:605-614, 2006. 
CAMARGO, O.A. \& ALLEONI, L.R.F. Compactação do solo e o desenvolvimento das plantas. Piracicaba, Escola Superior de Agricultura "Luiz de Queiroz"/Universidade de São Paulo, 1997. 132p.

COLLARES, G.L.; REINERT, D.J.; REICHERT, J.M. \& KAISER, D.R. Qualidade física do solo na produtividade da cultura do feijoeiro num Argissolo. Pesq. Agropec. Bras., 41:16631674, 2006.

DAO, T.H. Tillage system and crop residue effects on surface compaction of a paleustoll. Agron. J., 88:141-148,1996.

DIAS JÚNIOR, M.S. \& MIRANDA, E.E.V. Comportamento da curva de compactação de cinco solos da região de Lavras (MG). Ci. Agrotec., 24:337-346, 2000.

EKWUE, E.J. \& STONE, R.J. Organic matter effects on the strength properties of compacted agricultural soils. Trans. Am. Sci. Agric. Eng., 38:357-365, 1995.

EMPRESA BRASILEIRA DE PESQUISA AGROPECUÁRIA - EMBRAPA. Centro Nacional de Pesquisa de Solos. Manual de métodos de análise de solo. 2.ed. Rio de Janeiro, 1997. 212p.

EMPRESA BRASILEIRA DE PESQUISA AGROPECUÁRIA EMBRAPA. Centro Nacional de Pesquisa de Solos. Sistema brasileiro de classificação de solos. 2.ed. Rio de Janeiro, 2006. 399p.

GUPTA, S.C.; SHARMA, P.P. \& DEFRANCHI, S.A. Compaction effects on soil structure. Adv. Agron., 42:311-338, 1989.

JACOMINE, P.K.T.; CAVALCANTI, A.C.; PESSOA, S.C.P. \& SILVEIRA, C.O. Levantamento exploratório. Reconhecimento de solos do Estado de Alagoas. Recife, Embrapa/ Centro de Pesquisas Pedológicas/Sudene-DRN, 1975. 531p. (Boletim Técnico, 35)

KEMPER, W.D. \& ROSENAU, R.C. Aggregate stability and size distribution. In: KLUTE, A. (Ed.). Methods of soil analysis. Madison, American Society of Agronomy, 1986. Part 1. p.425-442. (Agronomy, 9)
KÖPPEN, W. \& GEIGER, R. Klimate der Erde. Gotha, Verlag Justus Perthes, 1928. Wall-map $150 \mathrm{~cm}$ x $200 \mathrm{~cm}$.

KRZIC, M.; BUHNER, C.E.; TESTE, F.; DOMPTER, L. \& RAHMAN, S. Soil properties influencing compactability of forest soils in British Columbia. Canadian J. Soil Sci., 84:219-236, 2004.

LINSTRON, M.J. \& VOORHEES, W.B. Responses of temperate crops in North America to soil compaction. In: SOANE, B.D. \& van OUWERKERK, C., eds. Soil compaction in crop production. London, Elsevier, 1994. p.265-286 (Developments in Agricultural Engineering, 2)

OJENIYI, S.O. \& DEXTER, A.R. Soil factor affecting the macrostrutured produced by tillage. Trans. Am. Sci. Agric. Eng., 22: 339-343, 1979.

PROCTOR, R.R. Fundamental principles of soil compaction. Eng. News Rec., 3:20-28, 1933.

RAPER, R.L. Agricultural traffic impacts on soil. J. Terramech., 42:259-280, 2005.

SAEG. Sistema para análises estatísticas. Versão 9.0. Viçosa, MG, Fundação Arthur Bernardes, 2005.

SILVA, A.J.N.; CABEDA, M.S.V. \& CARVALHO, F.G. Matéria orgânica e propriedades físicas de um Argissolo Amarelo Coeso sob sistemas de manejo com cana-de-açúcar. R. Bras. Eng. Agríc. Amb., 10:579-585, 2006.

SILVA, A.P.; LIBARDI, P.L. \& CAMARGO, O.A. Influência da compactação nas propriedades físicas de dois latossolos. R. Bras. Ci. Solo, 10:91-95, 1986.

STATSOFT STATISTICA. Data analysis software system. Version 6, 2001.

STONE, R.J. \& EKWUE, E.J. Maximum bulk density achieved during soil compaction as effected by the incorporation of three organic materials. Trans. Am. Sci. Agric. Eng., 36:1713-1719, 1993. 
